\title{
Aptitud reproductiva de toros criollo lechero tropical
}

\section{Breeding potential of criollo lechero tropical bulls}

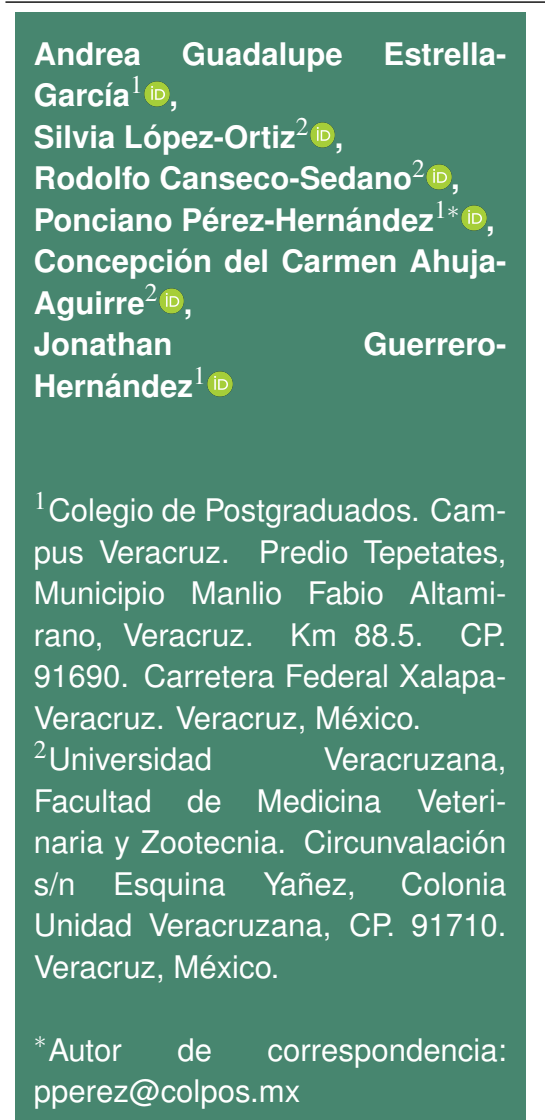

Artículo científico

Recibido: 28 de febrero 2021

Aceptado: 22 de septiembre 2021

Como citar: Estrella-García AG, López-Ortiz S, CansecoSedano R, Pérez-Hernández P, Ahuja-Aguirre CC, GuerreroHernández J (2021) Aptitud reproductiva de toros criollo lechero tropical. Ecosistemas y Recursos Agropecuarios Núm. Esp. II: e2930. DOI: 10.19136/era.a8nll.2930
RESUMEN. El ganado Criollo Lechero Tropical (CLT) posee características deseables para la ganadería tropical. Sin embargo, se desconoce el comportamiento reproductivo de toros CLT de diferentes edades durante las diferentes estaciones del año en clima cálido. En este estudio, ocho toros se asignaron según su edad a los siguientes grupos: 1) $\mathrm{G} 1(\mathrm{n}=5)$ : machos de $19.3 \pm 0.4$ meses y $260 \pm 9 \mathrm{~kg}$ de peso y 2$) \mathrm{G} 2(\mathrm{n}=3)$ : toros de 30.5 \pm 3.1 meses y $371 \pm 29 \mathrm{~kg}$ de peso. Durante un año se evaluó la libido en una escala de 0 a 10 puntos y se midieron las concentraciones séricas de testosterona. En las diferentes estaciones del año la libido presentó un puntaje de 8 a 10 para ambos grupos. Los toros del G2 tuvieron mayor concentración de testosterona $(P<0.05)$ y en ambos grupos ésta fue mayor en la mañana $(P<0.05)$. En conclusión, los toros CLT de ambos grupos mostraron un amplio potencial reproductivo durante las diferentes estaciones del año.

Palabras clave: Desempeño reproductivo, estación, edad, calidad seminal, semen.

ABSTRACT. Criollo Lechero Tropical (CLT) cattle have desirable characteristics for tropical cattle production. However, the reproductive performance of CLT bulls at different ages and in different seasons of the year has not been evaluated under tropical climate conditions. In this study, eight bulls were assigned to two age groups: 1) G1 $(n=5)$ : bulls at age $19.3 \pm 0.4$ months and $260 \pm 9 \mathrm{~kg}$ of live weight, and 2) G2 $(\mathrm{n}=3)$ : bulls at age $30.5 \pm 3.1$ months and $371 \pm 29 \mathrm{~kg}$ of live weight. Throughout one year, libido was evaluated in a 0 to 10-point scale and blood serum testosterone concentrations were determined. In the different seasons of the year, libido scored from 8 to 10 in both groups of bulls. Bulls from G2 had higher concentrations of testosterone $(P<0.05)$, and in both groups testosterone concentrations were higher during the morning hours $(\mathrm{P}<$ 0.05). In conclusion, CLT bulls from both groups showed a high reproductive potential throughout the seasons of the year.

Key words: Reproductive performance, season, age, semen quality, semen. 


\section{INTRODUCCIÓN}

En las regiones tropicales de México se practica comúnmente la ganadería de doble propósito, constituida en su mayoría por animales Bos indicus y cruzas Bos taurus $x$ Bos indicus y por una minoría de razas Bos taurus (González-Padilla et al. 2019). Sin embargo, la reproducción de los machos bovinos no ha recibido atención suficiente y la información al respecto es escasa, a pesar de que el $90 \%$ de los productores utilizan al toro para servir a las vacas mediante monta natural (Lassala et al. 2020), y a que entre 17 y $35 \%$ de los sementales utilizados presentan alguna alteración que reduce su capacidad reproductiva (Vejarano et al. 2005, Camacho et al. 2017). Aunque existe un bajo porcentaje de machos completamente estériles, los toros subfértiles pueden afectar la tasa de preñez y disminuir los ingresos del productor; lo que enfatiza la importancia de realizar la evaluación de la aptitud reproductiva de los toros antes de seleccionarlos como sementales (Paéz-Barón y Corredor-Camargo 2014).

Los toros realizan ciertas actividades para interactuar sexualmente con las hembras, generalmente la visión despierta el interés sexual inicial del toro y después investiga el estado fisiológico de las hembras para detectar su receptividad mediante el lamido y olfateo de la región perianal de las vacas (Chenoweth et al. 1996). Según Chenoweth et al. (1996), el comportamiento sexual de los toros comprende seis eventos importantes: 1) husmeo y lamido a la hembra para identificar las feromonas, 2) levantamiento de la cabeza y realización de flehmen, 3) erección del pene sin monta, con o sin movimiento pélvico, 4) goteo de fluido preeyaculatorio, 5) tentativa de monta, y 6) monta y eyaculación. Los toros con problemas reproductivos podrían no mostrar algunos de estos comportamientos y pasar inadvertidos, y por consiguiente, afectar la productividad de los hatos.

Los sementales de razas $B$. indicus son lentos para reaccionar sexualmente ante las vacas en estro y para efectuar la monta; en general, estas razas obtienen menores calificaciones en pruebas de libido que los toros de razas $B$. taurus (McCosker et al. 1989, Larsen et al. 1990). En un estudio realizado con toretes brahman y criollo limonero, se observó que al finalizar la prueba el $100 \%$ de los toros criollo limonero habían realizado por lo menos un servicio comparado con el $31 \%$ de los machos brahman (Ocanto et al. 1991). No obstante, el comportamiento sexual de la mayoría de los toros $B$. taurus es afectado por factores tales como la nutrición, el clima y el manejo, lo que dificulta utilizar sementales de este genotipo en hatos con poco manejo en clima cálido (Rodríguez et al. 1993).

Las razas bovinas criollas de América son descendientes del ganado $B$. taurus traído por los españoles durante la colonización. Estos bovinos criollos son manejados principalmente en el sistema de doble propósito (Severino-Lendechy et al. 2019), y se consideran como un recurso genético valioso por las características anatómicas, fisiológicas y biológicas que han desarrollado como resultado de la selección natural a la que se han sometido durante 500 años, que les permiten vivir bajo condiciones del clima cálido húmedo a subhúmedo y aprovechar eficientemente los forrajes con baja calidad nutricional, característicos de regiones con este clima (De-Alba 211).

Entre estas razas bovinas se encuentra el criollo lechero tropical (CLT), que posee alta fertilidad, calidad láctea y adaptación a las condiciones con clima cálido (Vilaboa et al. 2012), por lo que es una alternativa para aumentar la rentabilidad de la ganadería del trópico (Rosendo-Ponce y BecerrilPérez 2015, Parra-Cortés y Magaña-Magaña 2019). Se ha reportado que los toros criollos tienen mayor libido que toros cebú y holstein en el trópico (Madrid et al. 1995) y que las hembras criollas tienen mayor estabilidad fisiológica en condiciones climáticas adversas (Guerrero et al. 2011), lo que les permite un mayor equilibrio endocrino reflejado en un mejor comportamiento reproductivo (Severino-Lendechy et al. 2019). Sin embargo, hace falta evidencia de las capacidades reproductivas de los toros CLT y bajo el manejo que reciben en condiciones de los ranchos. Por lo anterior, en la presente investigación el objetivo fue evaluar la libido, características seminales y la concentración de testosterona de machos CLT en el trópico mexicano. 


\section{MATERIALES Y MÉTODOS}

\section{Ubicación geográfica y características del estudio}

El estudio se realizó en un hato bovino de raza CLT propiedad del Colegio de Postgraduados, Campus Veracruz, localizado a $19^{\circ} 16^{\prime} \mathrm{LN}$ y $96^{\circ} 16^{\prime} \mathrm{LO}$, a una altura de $20 \mathrm{msnm}$. El clima es AW1(w)(i')g según Köeppen, modificado por García (1988), descrito como cálido subhúmedo con temperatura promedio anual de $26.5{ }^{\circ} \mathrm{C}$ y precipitación anual de $1230 \mathrm{~mm}$, distribuidos entre los meses de mayo a octubre. El estudio se realizó a lo largo de un año y comprendió las cuatro estaciones: otoño (septiembre 22 a diciembre 20), invierno (diciembre 21 a marzo 19), primavera (marzo 20 a junio 20 ) y verano (junio 21 a septiembre 21$)$.

\section{Animales examinados}

Se utilizaron ocho toros seleccionados de un lote de 14 machos, con base en un examen que incluyó: 1) evaluación de la visión, considerando la respuesta a estímulos y que al caminar no se golpearan; 2) evaluación de aplomos, para confirmar que no presentaran cojera o alguna lesión y que no tuvieran desviaciones hacia los lados, adelante o atrás; 3) evaluación del prepucio, para confirmar que no tuviera lesiones ni que expulsara líquido purulento; 4) examen de próstata y vesículas seminales, para constatar que no existiera un crecimiento anormal o inflamación detectado por palpación rectal; 5) condición corporal (CC), para seleccionar animales con puntaje de 2.5 a 3.0 según la escala desarrollada por Wildman et al. (1982) que asigna calificaciones de 1 a 5 , donde 1 es un animal emaciado y 5 es obeso.

Los toros seleccionados se dividieron en dos grupos: Grupo $1(\mathrm{n}=5)$ : incluyó a machos con edad promedio de $19.3 \pm 0.4$ meses y peso promedio de $260 \pm 9 \mathrm{~kg}$, y Grupo $2(\mathrm{n}=3)$ : comprendió a toros con edad y peso promedio de $30.5 \pm 3.1$ meses y $371 \pm 29 \mathrm{~kg}$, respectivamente.

\section{Alimentación y manejo de los animales}

Durante el estudio, los animales permanecieron en potreros con las gramíneas Megathyrsus maximus, Brachiaria mutica, Cynodon nlemfluensis y gramas nativas del género Paspalum, manejados con una carga animal de 2 UA ha ${ }^{-1}$. Debido a la importancia de estudiar a los animales sin alterar su manejo y condiciones ambientales en que se encontraban, no se proporcionó suplementación, no se desparasitaron contra endo ni ectoparásitos, ni se aplicó ningún medicamento durante el estudio.

\section{Evaluación de la libido, conductas sexuales y de cortejo}

A los ocho toros se les evaluó la libido cada 60 d durante un año; las evaluaciones se iniciaron en el mes de septiembre, y a partir de ahí se asignaron a cuatro estaciones del año (otoño, invierno, primavera y verano). En cada evaluación, dos vacas que presentaban cuerpo lúteo se sincronizaron con $20 \mathrm{mg}$ de $\mathrm{PGF}_{2} \alpha$ (Lutalyse, Pfizer Inc. EU) tres días antes de la prueba. El examen se realizó entre las 9:00 y 11:30 h y se utilizó un orden aleatorio para el ingreso de los toros. Cada toro se colocó con una vaca en celo sujeta en un corral independiente durante 10 minutos, mientras los otros toros permanecieron en espera en potreros aislados. La libido se evaluó según la escala recomendada por Chenoweth (1986), que consiste en asignar calificaciones de 0 (no interés sexual) a 10 (dos o más servicios seguidos por interés sexual incluyendo montas, intentos de montas o servicios posteriores).

Las conductas de cortejo registradas fueron: 1) Olfateos: el toro olfateó la región genital de la hembra; 2) Flehmen: acción del macho a través de la obturación del labio superior para fijar hormonas en los receptores del órgano vomeronasal o de Jacobson e identificar secreciones feromonales de índole sexual; 3) Lamidos: el toro lamió la región genital de la hembra; y 4) Topeteo: el macho topeteó de forma no violenta a la vaca con la intención de llamar su atención.

Las conductas sexuales registradas fueron: 1) Intento de monta: movimientos de monta en los que el toro no logró apoyarse sobre la grupa de la hembra; 2) Monta: el toro se apoyó sobre la grupa de la hembra sin eyacular, aún cuando penetró a la hembra; 3) Monta completa: el toro montó a la hembra, ésta se quedó completamente quieta y el macho penetró y 
eyaculó, inferido por el movimiento de propulsión del semental.

\section{Características seminales}

De cada animal y en cada estación del año, excepto otoño, se recolectó una muestra de semen mediante electroeyaculador. Inmediatamente después de recolectado el semen se determinó su volumen mediante observación directa en el tubo de recolección, y se determinó la motilidad espermática en masa (MM) e individual (MI). Para determinar la MM se colocó una gota de $10 \mu \mathrm{L}$ de semen sobre un portaobjetos precalentado a $37^{\circ} \mathrm{C}$, se observó en un microscopio de contraste de fases (10x y 40x) y se le asignó un valor de 0 a $100 \%$. Para determinar la $\mathrm{Ml}$ se colocó una gota de $10 \mu \mathrm{L}$ de semen entre un portaobjetos y un cubreobjetos precalentados a $37^{\circ} \mathrm{C}$, se observó la motilidad rectilínea progresiva con microscopio de contraste de fases (10x y 40x) y se le asignó un valor de 0 a 100\%. La concentración de espermatozoides en el eyaculado se determinó con un hemocitómetro, para lo cual se diluyeron $10 \mu \mathrm{L}$ de semen en $2 \mathrm{~mL}$ de solución formol salina, se llenaron dos cámaras del hemocitómetro y se contó el número de espermatozoides presentes en cinco cuadros grandes de cada cámara usando un microscopio de contraste de fases (40x); el total de células contadas se multiplicó por 106 para obtener el número de espermatozoides por mililitro de semen. Para determinar la morfología espermática, se diluyeron $20 \mu \mathrm{L}$ de semen en $1 \mathrm{~mL}$ de solución formol salina y se analizaron según los criterios descritos por Blom (1973), usando un microscopio de contraste de fases (1000x); se evaluaron 400 células y se registró el porcentaje de células anormales.

\section{Concentraciones plasmáticas de testosterona}

Los mismos días en que se evaluó la libido, de cada animal se recolectaron $5 \mathrm{~mL}$ de sangre de la vena coccígea en tubos al vacío adicionados con anticoagulante EDTA (Etilen-Diamin-Tetra-Acetato). Se recolectó una muestra de sangre cada $30 \mathrm{~min}$ de 07:00 a 9:00 h y de 19:00 a 21:00 h. El muestreo se realizó en estos horarios porque la concentración de testosterona tiene un ritmo circadiano y tiende a ser más elevada por la mañana que por la noche. En los 30 minutos siguientes a su recolección, la sangre se centrifugó a $2000 \mathrm{~g}$ durante $15 \mathrm{~min}$ para separar el suero, mismo que fue conservado en microtubos de $2 \mathrm{~mL}$ a $-20{ }^{\circ} \mathrm{C}$ hasta su análisis. La concentración de testosterona se determinó mediante radioinmunoensayo en fase sólida en el laboratorio de la Universidad Nacional Autónoma de México, utilizando el kit CoatA-Count Testosterona Total (Diagnostic Products Corporation) y un contador de rayos gamma (AOKFIELD modelo 1996).

\section{Análisis estadístico}

Se utilizó estadística descriptiva para caracterizar la calificación de la libido, características seminales, concentración de testosterona, conductas sexuales y de cortejo, así como la frecuencia de las montas realizadas.

\section{RESULTADOS}

Durante el estudio, la temperatura y precipitación promedio mensual por época que se registraron fueron de $21.9 \pm 1.0^{\circ} \mathrm{C}$ y $0.25 \pm 0.2 \mathrm{~mm}$ en otoño, $22.6 \pm 1.9^{\circ} \mathrm{C}$ y $7.06 \pm 3.2 \mathrm{~mm}$ en invierno, $27.1 \pm 0.6^{\circ} \mathrm{C}$ y $109 \pm 13.3 \mathrm{~mm}$ en primavera y 27.8 $\pm 0.2^{\circ} \mathrm{C}$ y $55.7 \pm 17.5 \mathrm{~mm}$ en verano. La puntuación obtenida en la prueba de libido en los toros jóvenes (G1) fue de 9 a 10 y en los toros adultos (G2) de 8 a 9 (Tabla 1).

El comportamiento sexual de los toros se valoró en el rango de 6.3 a 6.7 veces en $\mathrm{G} 1$ y de 3.7 a 5.3 veces en G2 (Tabla 1). Los toros del G1 mostraron de 1.5 a 2.3 intentos de monta, 2.2 a 3.8 montas y 1.8 a 2.1 montas completas, mientras que los toros del G2 tuvieron 1.2 a 2.0 intentos de montas, 1.2 a 2.2 montas y 1.2 a 1.7 montas completas (Tabla 1).

Los machos de G1 manifestaron entre 7.2 a 9.5 conductas de cortejo y los de G2 entre 4.7 a 8.7, a través de todas las épocas evaluadas (Tabla 2). Las manifestaciones de cortejo de los toros en $\mathrm{G} 1$ fueron 2.2 a 3.1 olfateos, 2.7 a 3.2 flehmen y 1.3 a 3.5 lamidos, mientras que los del G2 mostraron 2.0 a 2.2 olfateos, 1.5 a 2.3 flehmen y 1.0 a 4.7 lamidos. 
Tabla 1. Libido y actividades sexuales de machos criollo lechero tropical menores de 30 meses (G1) y mayores de 30 meses de edad (G2) en el trópico suhúmedo de México.

\begin{tabular}{|c|c|c|c|c|}
\hline \multirow{2}{*}{ Grupo } & \multicolumn{4}{|c|}{ Época del año } \\
\hline & Otoño & Invierno & Primavera & Verano \\
\hline \multicolumn{5}{|c|}{ Edad (meses) ${ }^{\star}$} \\
\hline G1 & $19.3 \pm 0.4$ & $21.6 \pm 0.2$ & $24.8 \pm 0.4$ & $27.8 \pm 0.4$ \\
\hline G2 & $30.5 \pm 3.1$ & $33.3 \pm 3.1$ & $36.5 \pm 3.1$ & $39.2 \pm 3.1$ \\
\hline \multicolumn{5}{|l|}{ Libido } \\
\hline G1 & 9 & 10 & 9 & 9 \\
\hline G2 & 8 & 9 & 9 & 9 \\
\hline \multicolumn{5}{|c|}{ Conductas sexuales } \\
\hline G1 & $6.3 \pm 0.8$ & $6.6 \pm 0.6$ & $6.7 \pm 0.7$ & $6.4 \pm 0.7$ \\
\hline G2 & $3.7 \pm 0.2$ & $4.3 \pm 0.2$ & $5.3 \pm 0.8$ & $5.0 \pm 0.4$ \\
\hline \multicolumn{5}{|c|}{ Intento de monta } \\
\hline G1 & $2.3 \pm 0.4$ & $2.2 \pm 0.4$ & $1.5 \pm 0.3$ & $1.5 \pm 0.3$ \\
\hline G2 & $1.2 \pm 0.2$ & $1.5 \pm 0.3$ & $1.5 \pm 0.3$ & $2.0 \pm 0.0$ \\
\hline \multicolumn{5}{|l|}{ Monta } \\
\hline G1 & $2.2 \pm 0.7$ & $2.3 \pm 0.2$ & $3.8 \pm 0.9$ & $2.6 \pm 0.9$ \\
\hline G2 & $1.3 \pm 0.3$ & $1.2 \pm 0.6$ & $2.2 \pm 0.2$ & $1.5 \pm 0.3$ \\
\hline \multicolumn{5}{|c|}{ Montas completas } \\
\hline G1 & $1.8 \pm 0.3$ & $2.1 \pm 0.3$ & $1.7 \pm 0.3$ & $1.8 \pm 0.5$ \\
\hline G2 & $1.2 \pm 0.6$ & $1.7 \pm 0.2$ & $1.7 \pm 0.2$ & $1.5 \pm 0.3$ \\
\hline
\end{tabular}

Tabla 2. Conducta de cortejo de machos criollo lechero tropical menores de 30 meses (G1) y mayores de 30 meses de edad (G2) en el trópico suhúmedo de México.

\begin{tabular}{lcccc}
\hline \multirow{2}{*}{ Grupo } & \multicolumn{4}{c}{ Época del año } \\
\cline { 2 - 5 } & Otoño & Invierno & Primavera & Verano \\
\hline Conductas de cortejo & & & \\
G1 & $7.2 \pm 1.2$ & $7.3 \pm 1.1$ & $7.9 \pm 1.8$ & $9.5 \pm 0.9$ \\
G2 & $4.7 \pm 0.6$ & $4.8 \pm 0.4$ & $8.7 \pm 2.0$ & $7.5 \pm 1.6$ \\
Olfateos & & & \\
G1 & $3.1 \pm 0.9$ & $2.8 \pm 0.5$ & $2.3 \pm 0.6$ & $2.2 \pm 0.5$ \\
G2 & $2.2 \pm 0.2$ & $1.8 \pm 0.2$ & $2.0 \pm 0.3$ & $2.0 \pm 0.5$ \\
Flehmen & & & \\
G1 & $3.1 \pm 0.5$ & $2.7 \pm 0.9$ & $2.9 \pm 0.7$ & $3.2 \pm 0.9$ \\
G2 & $1.5 \pm 0.3$ & $1.7 \pm 0.2$ & $2.0 \pm 0.6$ & $2.3 \pm 0.5$ \\
Lamido & & & \\
G1 & $1.3 \pm 0.3$ & $1.7 \pm 0.4$ & $2.6 \pm 1.0$ & $3.5 \pm 0.6$ \\
G2 & $1.0 \pm 0.3$ & $1.3 \pm 0.2$ & $4.7 \pm 1.7$ & $2.2 \pm 0.4$ \\
\hline * Media \pm desviación estándar. Otoño = septiembre 22 a diciem- \\
bre 20, Invierno = diciembre 21 a marzo 19, Primavera = marzo \\
20 a junio 20, Verano = julio 21 a septiembre 21.
\end{tabular}

El tiempo medido entre dos montas completas fue $5.32 \pm 0.25$ min para toros del G1 y $6.17 \pm 0.17$ min para el G2. El grupo 1 presentó hasta cuatro montas completas en 10 min (Tabla 3). El porcentaje de toros con tres a cuatro montas en 10 minutos fue $62.6 \%$ en $\mathrm{G} 1$ y $58.4 \%$ en G2 (Tabla 3).
El volumen del eyaculado estuvo en el rango de 3.8 a $7.8 \mathrm{~mL}$, observándose en ambos grupos un comportamiento similar durante las estaciones del año; la mayor concentración espermática se encontró en ambos grupos de toros en verano (Tabla 4). La motilidad en masa fue del 88 al $90 \%$ en G1 y del 75 al $80 \%$ en G2. El mayor porcentaje de espermatozoides anormales en ambos grupos de toros se presentó en invierno (14.2 \pm 1.0 en $\mathrm{G} 1$ y $17.3 \pm 2.0$ en G2).

Tabla 3. Montas completas durante 10 minutos (\%) de toros criollo lechero tropical menores de 30 meses (G1) y mayores de 30 meses de edad (G2) en el trópico suhúmedo de México.

\begin{tabular}{lcccc}
\hline \multirow{2}{*}{ Grupo } & \multicolumn{4}{c}{ Montas completas, \% } \\
\cline { 2 - 5 } & 1 & 2 & 3 & 4 \\
\hline G1 & 0 & 37.3 & 35.3 & 27.3 \\
G2 & 8.3 & 33.3 & 58.4 & 0 \\
\hline
\end{tabular}

Tabla 4. Características seminales de machos criollo lechero tropical menores de 30 meses (G1) y mayores de 30 meses de edad (G2) en el trópico subhúmedo de México.

\begin{tabular}{|c|c|c|c|c|}
\hline \multirow{2}{*}{ Grupo } & \multicolumn{4}{|c|}{ Época del año } \\
\hline & Otoño & Invierno & Primavera & Verano \\
\hline \multicolumn{5}{|c|}{ Volumen del eyaculado (mL) } \\
\hline G1 & - & $4.1 \pm 0.6$ & $4.2 \pm 1.2$ & $6.6 \pm 1.2$ \\
\hline G2 & - & $3.8 \pm 0.7$ & $4.5 \pm 0.5$ & $7.8 \pm 3.4$ \\
\hline \multicolumn{5}{|c|}{ Concentración seminal $\left(\times 10^{6} \mathrm{~mL}^{-1}\right)$} \\
\hline G1 & - & $660 \pm 110$ & $786 \pm 91$ & $962 \pm 113$ \\
\hline G2 & - & $593 \pm 122$ & $610 \pm 191$ & $877 \pm 298$ \\
\hline \multicolumn{5}{|c|}{ Motilidad en masa (\%) } \\
\hline G1 & - & $88 \pm 2$ & $89 \pm 1$ & $90 \pm 2$ \\
\hline G2 & - & $80 \pm 2$ & $75 \pm 7$ & $80 \pm 4$ \\
\hline \multicolumn{5}{|c|}{ Motilidad individual (\%) } \\
\hline G1 & - & $88 \pm 2$ & $89 \pm 1$ & $91 \pm 2$ \\
\hline G2 & - & $80 \pm 2$ & $75 \pm 8$ & $82 \pm 2$ \\
\hline \multicolumn{5}{|c|}{ Espermatozoides anormales (\%) } \\
\hline G1 & - & $14.2 \pm 1.0$ & $7.2 \pm 0.6^{a}$ & $5.2 \pm 0.9$ \\
\hline $\mathrm{G} 2$ & - & $17.3 \pm 2.0$ & $14.0 \pm 3.0^{b}$ & $2.7 \pm 0.7$ \\
\hline \multicolumn{5}{|c|}{$\begin{array}{l}\text { - No se tomaron datos en esta época. }{ }^{*} \text { Media } \pm \text { desviación están- } \\
\text { dar. Invierno }=\text { septiembre } 22 \text { a diciembre } 20, \text { Otoño }=\text { diciembre } \\
21 \text { a marzo } 19, \text { Primavera }=\text { marzo } 20 \text { a junio } 20, \text { Verano }=\text { julio } 21 \\
\text { a septiembre } 21 .\end{array}$} \\
\hline
\end{tabular}

Las concentraciones séricas de testosterona en toros jóvenes y adultos fueron más elevadas durante la mañana (7:00 a 9:00 h) que durante la noche (19:00 a 21:00 h) (Tabla 5). Numéricamente, los toros adultos tuvieron mayores concentraciones de testosterona que los jóvenes (Tabla 5). 
Tabla 5. Concentración plasmática de testosterona ( $\mathrm{ng} \mathrm{mL}^{-1}$ ) de toros criollo lechero tropical menores de 30 meses (G1) y mayores de 30 meses de edad (G2) en el trópico suhúmedo de México.

\begin{tabular}{|c|c|c|c|c|}
\hline \multirow{2}{*}{ Grupo } & \multicolumn{4}{|c|}{ Época del año } \\
\hline & Otoño & Invierno & Primavera & Verano \\
\hline \multicolumn{5}{|l|}{$07: 00 \mathrm{~h}$} \\
\hline G1 & - & $10.3 \pm 0.8$ & $11.3 \pm 1.3$ & $9.0 \pm 1.1$ \\
\hline G2 & - & $8.7 \pm 3.7$ & $12.9 \pm 2.3$ & $15.9 \pm 3.4$ \\
\hline \multicolumn{5}{|l|}{$09: 00 \mathrm{~h}$} \\
\hline $\mathrm{G} 1$ & - & $4.2 \pm 0.9$ & $6.12 \pm 1.1$ & $2.4 \pm 0.6$ \\
\hline G2 & - & $5.3 \pm 2.7$ & $9.6 \pm 3.4$ & $10.7 \pm 2.7$ \\
\hline \multicolumn{5}{|l|}{$19: 00 \mathrm{~h}$} \\
\hline G1 & - & $3.0 \pm 1.3$ & $1.4 \pm 0.1$ & $5.4 \pm 1.1$ \\
\hline G2 & - & $5.9 \pm 3.0$ & $10.0 \pm 3.6$ & $7.8 \pm 3.9$ \\
\hline \multicolumn{5}{|l|}{$21: 00 \mathrm{~h}$} \\
\hline G1 & - & $2.0 \pm 0.8$ & $3.9 \pm 1.4$ & $1.8 \pm 0.6$ \\
\hline G2 & - & $3.8 \pm 3.0$ & $7.2 \pm 2.8$ & $6.1 \pm 3.8$ \\
\hline
\end{tabular}

\section{DISCUSIÓN}

Los resultados obtenidos en la libido de los toros CLT de ambos grupos es similar a la de toros criollo limonero (Madrid et al. 1995) y superior a toros brahman (Molina et al. 2001). La mayor puntuación en libido de los toros del G1 concuerda con lo señalado en toros simmental (López et al. 1999). La libido mostrada por los toros de ambos grupos se considera como alta; esto es importante en particular para ranchos de zonas tropicales que manejan hatos bovinos con monta natural sin detección de estros (González-Padilla et al. 2019, Lassala et al. 2020). Los toros más jóvenes (G1) no fueron afectados por la dominancia de los toros de mayor edad (G2), aun cuando se observó jerarquía dirigida por un toro del G2, lo que difiere a lo reportado por López et al. (1999).

En general, los valores de las conductas observadas en los toros son superiores a los promedios de las conductas sexuales (3.29) y de cortejo (4.43) observadas en toros brahman (Molina et al. 2001). Otros autores han reportado las actividades de lamer y flehmen como las actividades más observadas en toros holstein (Brockett et al. 1994).

El $100 \%$ de los toros presentaron protrusión y goteo de líquido seminal y reaccionaron inmediatamente al estar en contacto con una vaca en celo, in- dicando que los toros CLT tienen un comportamiento similar a los toros criollo limonero (Madrid-Bury et al. 2011). La acción de topeteo no fue una actividad frecuente; sólo dos toros del grupo 1 topetearon a la vaca durante la primera prueba y no se observó agresión por los toros durante la prueba.

La respuesta de los toros en G1 (19 a 29 meses) coincide con la reportada por López et al. (1999) en dos grupos de toros Simmental (18 y 27 meses), donde el grupo de toros de menor edad fue más activo y menos afectado por la dominancia. El número de montas completas fue similar al reportado (1 a 4) en ganado Bos taurus y superior al observado (0 a 1) en Bos indicus; además, Bertram et al. (2002) indicaron una disminución en la actividad sexual de toros Bos indicus al interactuar con hembras sujetas durante la prueba.

Independientemente de la edad y las observaciones reportadas en toros de otras razas, los toros CLT evaluados tuvieron una conducta sexual adecuada, lo cual es importante para los hatos del trópico latinoamericano donde predominantemente se utilizan toros Bos indicus (González-Padilla et al. 2019), que tienen una menor libido (Ocanto et al. 1991). Los toros CLT alcanzaron calificaciones similares a las reportadas en otras razas criollas de toros Bos taurus manejados en clima cálido (Madrid-Bury et al. 2011), lo que indica su adaptación a los climas cálidos e implica un alto potencial de servicio de los toros CLT bajo dichas condiciones y manejo extensivo.

El valor del volumen del eyaculado obtenido en ambos grupos de toros CLT fue mayor al obtenido con toros criollo limonero (Madrid et al. 1995) y costeño con cuernos (Palmieri et al. 2004). En ambos grupos de toros la mayor concentración espermática se presentó en verano, la estación más cálida del año, lo cual podría ser resultado de la adaptación de estos animales al clima cálido del trópico. La concentración seminal en ambos grupos de toros fue similar a la de toros criollo limonero $(819.5 \pm 91.5$ a $1416.2 \pm 175.1$ mill $\mathrm{mL}^{-1}$ ) de la misma edad (Madrid et al. 1995) y menor a la de costeño con cuernos (Palmieri et al. 2004). La motilidad en masa e individual fueron altas en ambos grupos de toros y fueron mayores a las de toros criollo limonero (Madrid et al. 1995) y costeño 
con cuernos (Palmieri et al. 2004). El mayor porcentaje de espermatozoides anormales se presentó en invierno, probablemente porque en dicha época se tuvieron vientos intensos y la precipitación mensual por época fue menor, $(0.25 \pm 0.2 \mathrm{~mm}$ en otoño, 7.06 $\pm 3.2 \mathrm{~mm}$ en invierno, $109 \pm 13.3 \mathrm{~mm}$ en primavera y $55.7 \pm 17.5 \mathrm{~mm}$ ) lo que disminuyó la disponibilidad de forraje y pudo ser causa de estrés en los animales. El porcentaje de espermatozoides anormales fue menor al $20 \%$ y fue similar al obtenido en toros criollo limonero (Madrid et al. 1995) y menor al de toros costeño con cuernos (Palmieri et al. 2004)

Las concentraciones plasmáticas de testosterona en toros jóvenes y adultos obtenidas en el presente estudio durante la mañana $(7: 00$ a 9:00 h) y durante la noche (19:00 a 21:00 h) fueron similares a lo observado por Ramírez y Faccio (2000) y JiménezSeveriano et al. (2003).

\section{CONCLUSIONES}

Los toros CLT estudiados se caracterizaron por tener alta libido y buena calidad seminal, independientemente de la edad, lo cual indica su potencial para utilizarse en programas reproductivos. Los toros jóvenes (19 meses de edad) mostraron el mismo comportamiento sexual que los toros de mayor edad, aunque tuvieron menores concentraciones de testosterona. Estos resultados sugieren que los toros CLT a partir de los 19 meses de edad pueden tener un desempeño reproductivo similar que toros adultos.

\section{LITERATURA CITADA}

Bertram JD, Fordyce GM, McGowan MR, Jayawardhana JA, Fitzpatrick LA, Doogan VJ, De Faver J, Holroyd RG (2002) Bull selection and use in northern Australia. Serving capacity test. Animal Reproduction Science 71: 51-66.

Blom E (1973) The ultrastructure of some characteristics sperm defects and a proposal for a new classification of the bull spermiogram. Nordisk veterinaer medicin. 25: 383-391.

Brockett CC, Presicce GA, Foote RH (1994) Semen quality and behavior of Holstein bulls exposed to estradiolTreated Bulls for mounts. Journal Dairy Science 77: 124-131.

Camacho SJ, Ramírez GS, Aguilar MP. 2017. Capacidad reproductiva de sementales en fincas ganaderas de Costa Rica. Revista UTN 80: 46-50.

Chenoweth PJ (1986) Libido testing. In: Morrow DA (ed) Current therapy in theriogenology. Saunders Company. Philadelphia, USA. pp: 136-142.

Chenoweth PJ, Chase J, Thatcher M, Wilcox C, Larsen R (1996) Breed and others effects on reproductive traits and breeding soundness categorization in young beef bulls in Florida. Theriogenology 46: 1159-1170.

De-Alba J (2011) Los Criollos Lecheros Tropicales. En: de-Alba-Martínez J (ed) El libro de los bovinos criollos de América. Biblioteca Básica de Agricutura - Colegio de Postgraduados. Ediciones Papiro Omega. México. pp: 91-156.

García E (1988) Modificación a sistemas de clasificación de Köppen (para adaptarlo a las condiciones de la Republica Mexicana). Universidad Autónoma de Chapingo. México. 194p.

González-Padilla E, Lassala A, Padernera M, Gutiérrez CG (2019) Cow-calf management practices in México: Farm organization and infrastructure. Veterinaria México OA 6(3). DOI: 10.22201/ fmvz.24486760e.2019. 3.677. 
Guerrero HLJ, Pérez-Hernández P, López-Ortiz S, Montiel-Palacios F, Estrella-García A, Aluja-Aguirre C (2011) Tasa de ovulación sincronizada con $\mathrm{PGF}_{2} \alpha$ y dinámica folicular en vacas criollas lechero Tropical. Archivos de Zootecnia 60: 1335-1338.

Jiménez-Severiano H, Quintal-Franco J, Vega-Murillo V, Zanella E, Wehrman ME, Lindsey BR, Melvin EJ, Kinder JE (2003) Season of the year influences testosterone secretion in bulls administered luteinizing hormone. Animal Science 81: 1023-1029.

Larsen RE, Littell R, Rooks E, Adams EL, Falcon C, Warnick AC (1990) Bulls influences in conception percentage and calving date in Angus, Hereford, Brahman and Senepol. Theriogenology 34: 549-568.

Lassala A, Hernández-Cerón J, Pedernera M, González-Padilla E, Gutiérrez CG (2020). Cow-calf management practices in México: Reproduction and breeding. Veterinaria México OA 7(1). DOI: 10.22201/fmvz.24486760 e.2020.1.839.

López H, Orihuela A, Silva E (1999) Effect of the presence of a dominant bull on performance of two age group bulls in libido tests. Animal Behaviour Science 65: 13-20.

Madrid N, Urdaneta R, Bracho I, Labbé S, Aranguren J (1995) Desarrollo corporal, circunferencia escrotal y características seminales de toros Criollo Limonero en Venezuela. Revista Argentina de Producción Animal 15: 967-968.

Madrid-Bury N, González-Satagnaro C, Aranguren-Méndez JA, Yañez F, Quintero-Moreno A (2011) Comportamiento sexual en criollo limonero. Revista Facultad de Agronomía (LUZ) 28: 505-513.

McCosker TH, Turner AF, Mc Cool CJ, Post TB, Bell K (1989) Brahman bull fertility in a Nort Australian rangeland herd. Theriogenology 32: 285-300.

Molina R, Bolaños I, Galina C, Pérez E, Paniagua G, Estrada S (2001) Sexual behaviour of Zebu bulls in the humid tropics of Costa Rica: single versus multiple sire groups. Animal Reproduction Science 64: 139-148.

Ocanto D, Linares T, Patiño A, Ramos C, Escobar S (1991) Conducta sexual en machos Criollo Limonero y Brahmán hasta la pubertad. Zootecnia Tropical 11: 55-70.

Paéz-Barón EM, Corredor-Camargo ES (2014) Evaluación de la aptitud reproductiva del toro. Ciencia y Agricultura 11: 49-59.

Palmieri R, Suárez D, Espitia A, González M, Prieto E (2004) Variables seminales de toros criollos colombianos costeños con cuernos y Romosinuano. Revista MVZ Córdoba 9: 381-385.

Parra-Cortés RI, Magaña-Magaña MA (2019) Características técnico-económicas de los sistemas de producción bovina basados en razas criollas introducidas en México. Ecosistemas y Recursos Agropecuarios 6: 535547.

Ramírez I, Faccio G (2000) Determinación directa de testosterona en suero sanguíneo de machos bovinos. Revista de Agronomía 7: 255-263.

Rodríguez C, Galina C, Gutiérrez C, Navarro R, Piccinalli R (1993) Evaluación de la actividad sexual de los toros cebú bajo condiciones de empadre múltiple con hembras sincronizadas. Ciencias Veterinarias 15: 41-49.

Rosendo-Ponce A, Becerril-Pérez CM (2015) Avance en el conocimiento del bovino Criollo Lechero Tropical de México. Ecosistemas y Recursos Agropecuarios 2: 233-243.

Severino-Lendechy VH, Perezgrovas-Garza RA, Montiel-Palacios F, Vilaboa-Arroniz J, Muñoz-González JC, Piñeiro-Vázquez AT (2019) Caracterización del nivel tecnológico y edad al primer estro de hembras Criollo Lechero Tropical. Ecosistemas y Recursos Agropecuarios 6: 353-359. 
Vejarano OA, Sanabria LRD, Trujillo LGA (2005) Diagnóstico de la capacidad reproductiva de toros en tres ganaderías de tres municipios del alto Magdalena. Revista MVZ Córdova 10: 648-662.

Vilaboa AJ, Quirós Madrigal OJ, Díaz-Rivera P, Zetina-Córdoba P (2012) Situación del bovino Criollo Lechero Tropical (CLT) en México, Nicaragua y Costa Rica. Archivos de Zootecnia 61: 31-39.

Wildman EE, Jones GM, Wagner PE, Boman RL, Trout JR, Lesch TN (1982) Dairy cow body condition scoring system and its relationship to selected production characteristics. Journal Dairy Science 65: 485-497. 